\title{
Efficient Algorithm for Railway Tracks Detection Using Satellite Imagery
}

\author{
Ali Javed \\ Department of Software Engineering, U.E.T Taxila \\ Taxila, Pakistan \\ Email: ali.javed@uettaxila.edu.pk \\ Khuram Ashfaq Qazi \\ Department of Software Engineering, U.E.T Taxila \\ Taxila, Pakistan \\ Email: gemni1987@yahoo.com \\ Muazzam Maqsood \\ Department of Software Engineering, U.E.T Taxila \\ Taxila, Pakistan \\ Email: muazzammaqsood@yahoo.com \\ Khurram Ali Shah \\ Department of Software Engineering, U.E.T Taxila \\ Taxila, Pakistan \\ Email: syedkhuram84@gmail.com
}

\begin{abstract}
Satellite imagery can produce maps including roads, railway tracks, buildings, bridges, oceans, lakes, rivers, etc. In developed countries like USA, Canada, Australia, Europe, images produced by Google map are of high resolution and good quality. On the other hand, mostly images of the third world countries like Pakistan, Asian and African countries are of poor quality and not clearly visible. Similarly railway tracks of these countries are hardly visible in Google map. We have developed an efficient algorithm for railway track detection from a low quality image of Google map. This would lead to detect damaged railway track, railway crossings and help to schedule/divert locomotive movements in order to avoid catastrophe.
\end{abstract}

Index Terms - Efficient, Detection, Locomotive and catastrophe

\section{INTRODUCTION}

Railway track detection is a very important task in image processing because there are so many lives are associated with it. If driver could not see another train then the many lives can be on stake. In developing countries like Pakistan, railway tracks are not in its best of shape, there are many reasons behind that phenomenon, terrorism attacks are the major reason behind it, floods and many other natural disasters. There is not a proper system to handle and look after the railway system and if it exists even then nobody is fulfilling its duty to take care of those tracks.
The only thing which happens due to this problem is that railway ministers are criticized badly and most of the time this brings changes at the very top level of the ministry. This can leads into political instability in the country.

Google maps are the best facility available so far for the detection of railway tracks but there are lot of issues in usage of Google maps. The main issue is that free Google service does not provide the detection of railway tracks easily. Secondly quality of images provided by free Google service is not very upright resulting mostly in the failure for the detection of railway tracks.

Google provides a very high quality for European countries like Canada, U.K, USA, France, Germany, Australia and japan.

That's why railway tracks can be very easily detected from high quality images and second reason for the easy detection is that railway tracks are very well maintained in European countries unlike most of the Asian countries.

In this research paper we will develop an efficient algorithm for the detection of railway tracks from the Google images solving the above mentioned issues.

This research can be used for many purposes like detection of the broken portions of the railway tracks, obstacles can be easily detected using this algorithm.

Train drivers can use this algorithm to detect the obstacles and broken parts and can change their path in 
advance. This research can also be extended in academic, scientific and educational purposes [1].

Digital image processing basically depends on some core steps like acquisition, pre-processing, object detection and then the most important part which is object recognition.[1] Mat Lab is used for the development tool. This simulation gives some extraordinary results for the detection and recognition of railway tracks.

This research paper is divided into seven portions, $1^{\text {st }}$ portion covers the introductory portion, second portion covers the literature review related to this research. Third portion explains the methodology used in this kind of systems and fourth portion covers the related work in this field.

Portion five covers the system architecture while $6^{\text {th }}$ portion explains the proposed system and the results of this research. Portion seven conclude this research followed by references.

\section{LITERATURE REVIEW}

The purpose of image enhancement lies in improving the human perception of information stored in the images. This results in providing a quality image as an input to other image processing methods. [2]

Getting an original information signal from a degraded image comes under the process of image restoration.[1] The basic aim of image restoration is to convert a degraded image into defects free image. Degradation is caused by a number of reasons including noise, blurred motion and poor lens focusing.

Segmentation is the process of dividing an image into separate regions or sub groups. The purpose of segmentation is to represent an image using meaningful information that should be simple and can be analyzed easily. Image Segmentation algorithms in image processing make use of two important characteristics of intensity values; these are discontinuity and similarity. [5]

Recognition refers to assigning labels to objects on the basis of defined descriptors. Pattern recognition through machine is a process that uses methods for allocating pattern to their relevant classes and this requires very little human effort.

\section{METHODOLOGY}

Some of the classical methods used for object detection and recognition are explained below.

\section{A. SVM (Support Vector Machine)}

This method analyzes different kind of data and then recognizes different patterns, these results further used to classify data and for the analysis purposes. A set of data is the input of the SVM and then this system predicts the results for each input data [2].
For the training purposes input is marked as belonging to one or two categories, SVM algorithm classify those input into one or the other category. This makes it less efficient due to time consumption in the training purposes [5].

\section{B. HAAR Transform}

HAAR transform is referred for color and gray level images. Haar wavelets and shifts are used. This is more or less like Fourier transform [9].

\section{SIFT (Scale Invariant Feature Transform)}

SIFT is an algorithm used for detection of objects based on several factors including coordinates, dimensions, intensity and scaling etc. This transform specifies some of the local features in a given image. [10]

Some of the applications of SIFT includes recognition of objects, mapping of robotics, stitched images, 3D Modeling, gesture recognition, Video tracking and frame matching [11]

\section{HOUGH Transform}

Hough Transform is a methodology used for extracting features which further helps in road tracking, line detection of fixed patterns etc. The aim of the method is to find out the faulty instances within a particular class of shapes and sizes. This finding procedure is based on a voting scheme. Voting is based on certain parameters which help in obtaining candidates for voting as local maxima.[17] These object candidates are obtained in an accumulator space that is developed by the algorithm of Hough Transform.[14]

Hough transform is basically used to detect lines and in our proposed system this property of Hough transform is used to detect the railway tracks.

\section{E. Hit Miss Transform}

HIT MISS transform is basically used in detection of objects on a small scale. It helps in pointing out the positions where patterns have occurred in the given image. The hit-or-miss transform also helps in identifying the terminal points of a line, so that we can easily remove false branches from both ends. [12]

\section{RELATED WORK}

Fatih Kaleli and Yusuf Sinan Akgul [14] used Dynamic programming in railroad environments to track space in front of the train and tracks are extracted using specially designed tracking algorithms. [17] Dynamic programming in this context computes the finest path requiring minimum cost in extracting the tracks. The projected algorithm extracts the rails in both left and right directions simultaneously using dynamic programming [14]. A camera was located in front of a train for extraction. 
Ross, R. [13] worked on Track selective localization which involves usage of a mono focal video camera which helps in improving the quality of localization. Algorithm uses recursive estimation for the camera pictures. [13] The results of estimation are used for turnout detection. As compared to GPS/INS bends and turnouts can be detected earlier. In this method, recursive estimation is used for locating tracks in the images and to calculate the geometry of the tracks.[16]

$\mathrm{Zu}$ Whan Kim [17] for detecting moving objects through a moving camera an efficient algorithm is proposed. The detection of moving object is accomplished through the following two criteria.

- Estimation of the camera's ego motion

- Detecting those aspects which have inconsistent motion compared with the motion of camera

\section{SYSTEM ARCHITECTURE}

The first phase in visioning system is the image acquisition. After acquiring image, it can be processed through various image processing methods for performing different tasks required for vision systems. However, the required tasks may not be achieved if the obtained image is not a satisfactory one. The Architecture of the system is discussed below.

System Architecture is shown in Figure 3. The most important step is image acquisition of required tracks. We obtain images from the Google maps and then all the processing is done on this image.

Figure 1 shows the noisy Image of railway track. When we processed that image the required informative portion vanished. Figure 2 shows the blur image of railway track. Same results achieved when we applied our algorithm to this image.

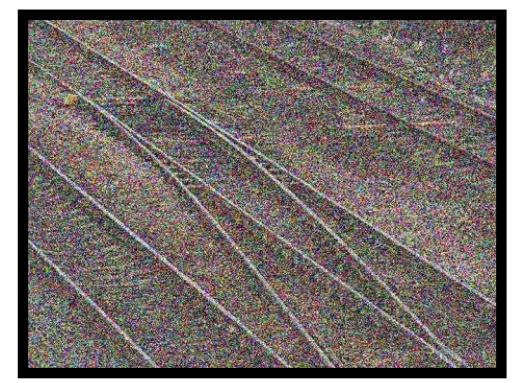

Figure 1 Noisy Train Track Image

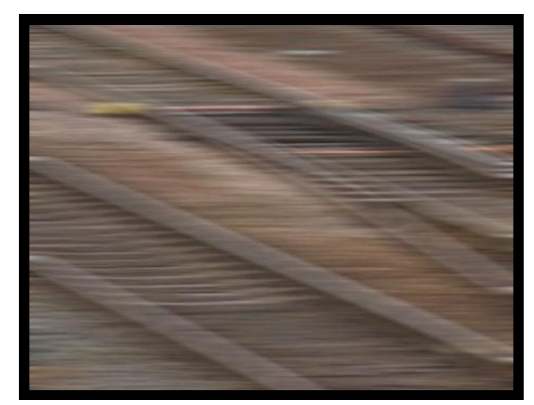

Figure 2 Blur Train Image
Due to this reason, Google Maps Images of railway tracks from the third world countries are used. The Designed System consists of 7 major steps as show in the Figure 3. The First step is image acquisition. Image is obtained from the Google Map source.

The obtained image is enhanced in the preprocessing phase. In the Preprocessing phase, the input image is undergoes Noise Removal Phase. The Noise Removed image is sharpened.

The resultant sharpened image is converted from color image to gray level image for further processing.

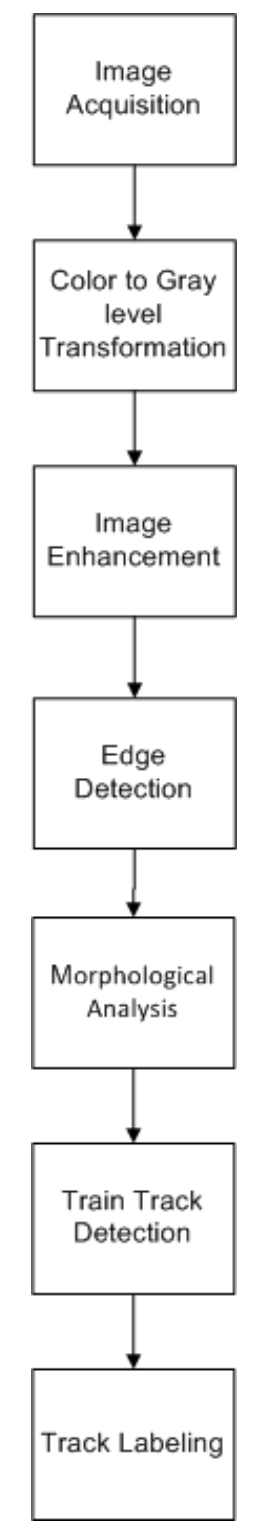

Figure 3 System Architecture

The $\mathrm{C} 2 \mathrm{G}$ converted image is processed for edge detection. Different edge detection algorithms are tested like Canny Edge detection, Robert Cross and Log Transform. The edge detected image is further processed for railway track detection which is our main motto. Morphological analysis is applied to image. In Morphological Analysis we applied erosion and dilation. 
After Morphological Analysis, the railway track is detected. The detected track is marked and labeled.

\section{PROPOSED METHODOLOGY}

The images of the railway tracks captured from the Google Maps are of not good quality. We have to detect the train track from the images that are gathered from Google Maps. The methodology used is basically based on our proposed algorithm. The image obtained from the Google Maps is shown as in figure 4.

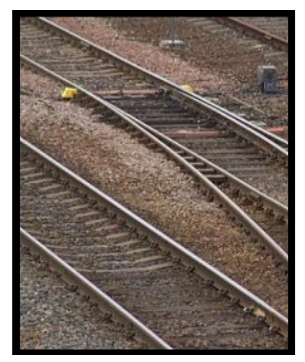

Figure 4 Image of railway track

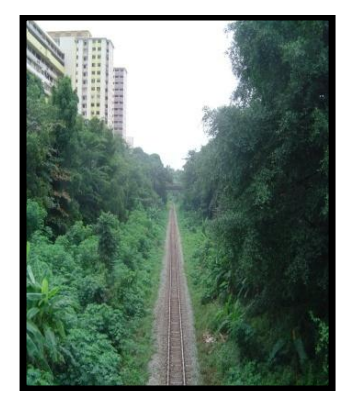

Figure 5 Image of railway track

The images are not clear as shown in the figures because these images are of third world countries. The steps involved in the railway track detection are explained below,

\section{A. PREPROCESSING}

Preprocessing is the major step in the processing of digital images as this enhances the quality of input / acquired image. In pre-processing three steps are performed.

The First Step is converting the image from $\mathrm{C} 2 \mathrm{G}$ level. Means image is converted into gray scale level. This converted gray scale image is shown in figure 6 given below.

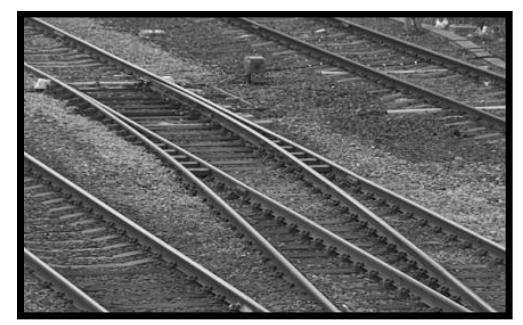

Figure 6 C2G Converted Image
The second step is noise removal. The Google Maps images are best quality images but of third world countries like Pakistan, images are noisy and blur. Averaging filtered produced the best result image for noise removal as shown in figure 7.

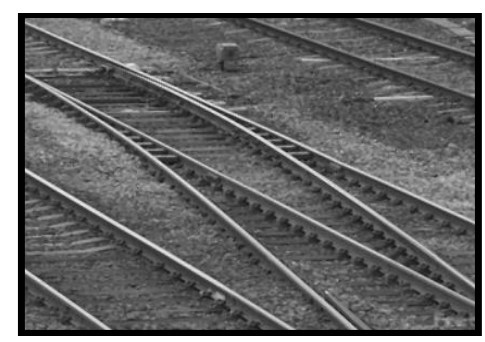

Figure 7 Noise Removal

The noisy removed image becomes blurring and needs enhancement by means of sharpening. The reason for sharpening is that while noise removal some information becomes blur. In order to regain the information the image is sharpened.

\section{B. EDGE DETECTION}

The sharpened image is then processed further for track detection. The main basic step for track detection is edges detection of railway track which were processed further for tracking and labeling. The reason is that the railway track has lines and it is better to step forward through this. Many algorithms have been developed for edges detection. The first tested algorithm was canny edge detection, when applied; The Output image of canny edge detection is shown as

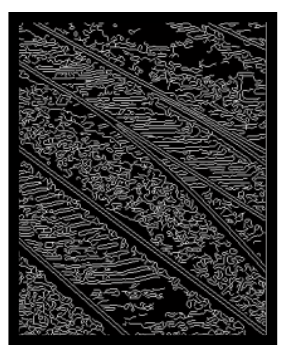

Figure 8 Canny Edge Detection

Above figure shows that results are noisy and not informative. So we tested other algorithms.

When we applied Robert Cross algorithm, the results were little better than canny edge detection but not according to the requirements. We achieved some better results but not according to requirements as show in figure.

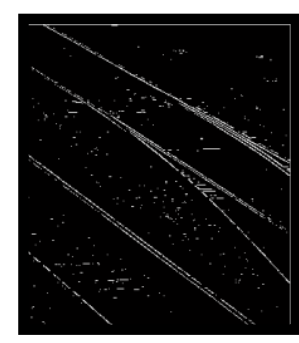

Figure 9 Robert Cross 
After observing these results we moved to test the Zero Cross Edge detection algorithm. But it went in vain as the figure given below clearly shows the noise and blurriness.

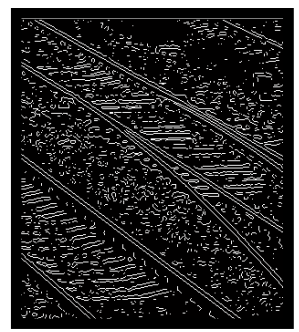

Figure 10 Zero Cross

Observing all results, finally applied the Sobel Algorithm. The results were amazing and we got the image according to the requirements.

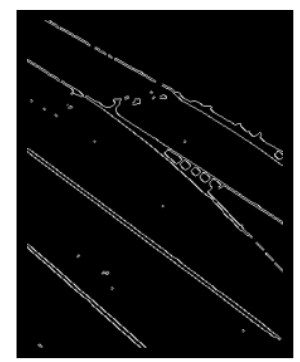

Figure 11Sobel Algorithm

\section{MORPHOLOGICAL ANALYSIS}

The output image obtained after the Sobel edge detection contains the required informative portion. We then applied the morphological analysis to output image obtained. It has two basic steps.

- Apply Erosion to Image.

- Apply Dilation to Output of Erosion

For Erosion we used the Square Filter with value 4 provided by the Matlab. The image obtained from Erosion is processed to Dilation having Diamond Filter with value 3 provided by Matlab. This technique is called Opening. The final result contains the lines filled as shown below,

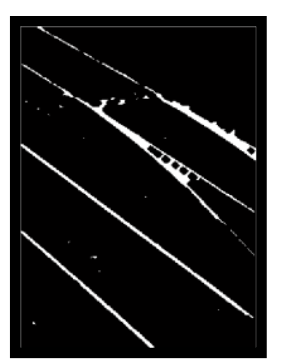

Figure 12 Morphological Analysis

\section{RAILWAY TRACK TRACKING AND MARKING}

Then the resultant image was processed for contrast and histogram equalization. After that we have to finally recognize the Tracks. The track was recognized by using our self-sample lining technique. We have used the Plus Operator for marking the Track and this Operator was provided by the Google System. The Plus Operator in green color shown in the given below figure clearly shows the detected railway track.

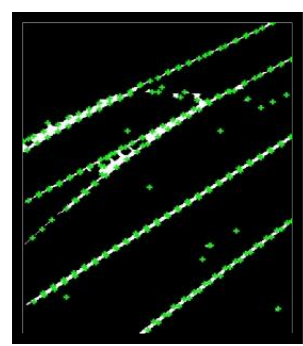

Figure 13 Track Detected

\section{CONCLUSION \& FUTURE WORK}

Algorithm developed in this research work is very efficient as shown by the results. It is very helpful to detect obstacles in railway tracks so that accidents can be avoided. It provides the solution of poor quality railway track images obtained by Google map. It can also be used for academic and scientific research. Another important aspect is that it can also be used in town planning, infrastructure extension and defence purposes. The proposed research work can be extended in many related areas such as real time monitoring of railway tracks, coordination methods between different units in locomotive safety field, etc.

\section{REFERENCES}

[1] Li, Y.-N.; Lu, Z.-M.; Niu, X.-M., ' 'Fast video shot boundary detection framework employing preprocessing techniques Image Processing”, IET pp. 121 - 134, June 2009.

[2] Zheng-ming Wang; Wei-wei Wang, "Fast and Adaptive Method for SAR Superresolution Imaging Based on Point Scattering Model and Optimal Basis Selection Image Processing", IEEE Transactions, pp. 1477 - 1486, July 2009.

[3] Jiuxiang Hu; Razdan, A.; Femiani, J.C.; Ming Cui; Wonka, P, "Road Network Extraction and Intersection Detection From Aerial Images by Tracking Road Footprints Geoscience and Remote Sensing", IEEE Transactions, pp. 4144 - 4157, Dec. 2007.

[4] Tupin, F.; Houshmand, B.; Datcu, M., "Road detection in dense urban areas using SAR imagery and the usefulness of multiple views Geoscience and Remote Sensing", IEEE Transactions, pp. 2405 - 2414, Nov 2002.

[5] Jing-Hao Xue; Titterington, D.M, “t-Tests, F-Tests and Otsu's Methods for Image Thresholding Image Processing", IEEE Transactions pp. 2392 2396, Aug. 2011.

[6] Dawoud, A.; Kamel, M.S, "Iterative multimodel subimage binarization for handwritten character 
segmentation Image Processing", IEEE Transactions, pp. 1223 - 1230, Sept. 2004.

[7] Bovolo, F.; Bruzzone, L.; Carlin, L, 'A Novel Technique for Subpixel Image Classification Based on Support Vector Machine Image Processing', IEEE Transactions, pp. 2983 2999, Nov. 2010.

[8] Li Cheng; Minglun Gong; Schuurmans, D, 'Caelli, T.; Real-Time Discriminative Background Subtraction Image Processing', IEEE Transactions, pp. 1401 - 1414, May 2011.

[9] Chong Chen; Schonfeld, D.; "A Particle Filtering Framework for Joint Video Tracking and Pose Estimation Image Processing”, IEEE Transactions, pp. 1625 - 1634, June 2010.

[10] Xuemei Ding; Wenjing Kang; Jiwen Cui; Lei Ao; "Automatic extraction of road network from aerial images Systems and Control in Aerospace and Astronautics", ISSCAA 2006. 1st International Symposium, pp. 223, 19-21 Jan. 2006.

[11] Movaghati, S.; Moghaddamjoo, A.; Tavakoli, A.; "Road Extraction From Satellite Images Using Particle Filtering and Extended Kalman Filtering Geoscience and Remote Sensing”, IEEE Transactions, pp. $2807-2817$, July 2010.

[12] Sirmacek, B.; Unsalan, C.; "Building detection from aerial images using invariant color features and shadow information Computer and Information Sciences", ISCIS '08. 23rd International Symposium, pp. 1 - 5, 27-29 Oct. 2008.

[13] Long Ma; Chunheng Wang; Baihua Xiao; "Text detection in natural images based on multi-scale edge detetion and classification Image and Signal Processing (CISP)", 2010 3rd International Congress pp. 1961 - 1965, 16-18 Oct. 2010.

[14] Jiejing Zhou; Yunfei Liu; Peng Li; '’Research on Binarization of QR Code Image Multimedia Technology (ICMT)', 2010 International Conference, pp. 1 - 4, 29-31 Oct. 2010

[15] Ying-Li Tian; Lu, M.; Hampapur, A., "Robust and efficient foreground analysis for real-time video surveillance Computer Vision and Pattern Recognition", IEEE Computer Society Conference, pp. 1182 - 1187, vol 1, 20-25 June 2005.

[16] Shivanand, T.; Rahman, S.; Pillai, G, "Efficient and robust detection and recognition of objects in grayscale images Computational Intelligence and Computing Research (ICCIC)", 2010 IEEE International Conference, pp. 1 - 6, 28-29 Dec. 2010.

[17] Chandrappa, D.N.; Ravishankar, M.; RameshBabu, D.R, "Face detection in color images using skin color model algorithm based on skin color information Electronics Computer Technology
(ICECT)", 2011 3rd International Conference, pp: $254-258,8-10$ April 2011.

[18] Sathya, P.D.; Kayalvizhi, R, "Image segmentation using minimum cross entropy and bacterial foraging optimization algorithm Emerging Trends in Electrical and Computer Technology (ICETECT)", 2011 International Conference, pp. 500 - 506, 23-24 March 2011.

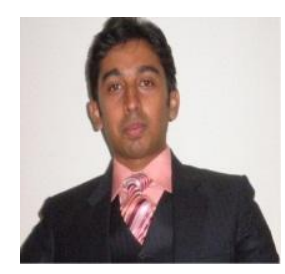

Engr. Ali Javed is a PhD Scholar University of Engineering \& Technology Taxila. He has been serving as an Assistant Professor in the Department of Software Engineering, University of Engineering and Technology Taxila, Pakistan. He has received his MS Computer Engineering degree from university of Engineering and Technology Taxila, Pakistan in February, 2010. He graduated from University of Engineering and Technology taxila in Software Engineering in September 2007. His areas of interest are Video Summarization, Digital Image Processing, Computer vision, Software Quality Assurance, Software testing, Software Requirements Analysis and Mobile application development.

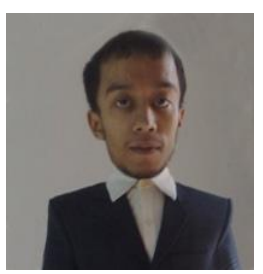

Engr. Khurram Ashfaq Qazi is MS Scholar in Department of Software Engineering at University of Engineering and Technology, Taxila, Pakistan. He has done his B.Sc Software Engineering from University of Taxila, Pakistan. He has a great research work on Noise Removal Algorithms in Image Processing. In 2008 he stayed in National Database and Registration Center to Study finger print or thumb impression image processing. In 2009 has worked in Software Organizations in R\&D Department. He has research work in Improving Quality of Software Products and improving development techniques. During 2010, he has worked in improving products quality relating to medical. During 2011 and interest in research work relating to Project Management, Advance Software technologies and Improving and Implementing Logical algorithms.

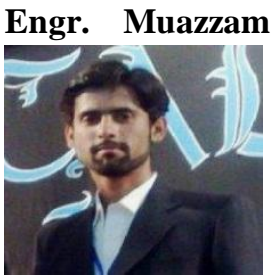

Maqsood is MS Scholar in Department of Software Engineering at University of Engineering \& Technology Taxila. He holds a Bachelor's degree in Software Engineering from University of Engineering \& Technology Taxila and has performed exceptional on 
his 4 year degree program. His core areas of interest are digital image processing, Software quality assurance, Software Project management and Video Summarization. Muazzam has been striving to bring innovations in the said fields through his research.

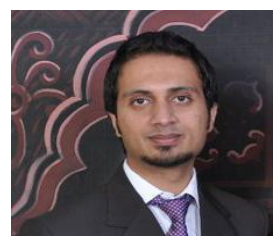

Engr. Khurram Ali Shah is MS Scholar in Department of Software Engineering at University of Engineering \& Technology Taxila. He has done his Bachelor's degree in Software Engineering from University of Engineering \& Technology Peshawar and has performed exceptional on his 4-year program. He has a keen interest in Software Project management, Software quality assurance and Computer Networks. Khurram has also worked in a software house in the department of Quality Assurance. His work experience is reflected in his research papers. One of his important researches includes IT project management where he has depicted the practical issues faced in the industry. 